\title{
Bayesian Reliability Analysis of Exponential Distribution Model under a New Loss Function
}

\author{
Haiping Ren ${ }^{\mathrm{a}}$ and Shigang $\mathrm{Chao}^{\mathrm{b}, *}$ \\ ${ }^{a}$ Teaching Department of Basic Subjects, Jiangxi University of Science and Technology, Nanchang, 330013, China \\ ${ }^{b}$ School of Mathematics and Computer Science, Yichun University, Yichun, 336000, China
}

\begin{abstract}
Loss function is an important content in Bayes statistical inference. The task of this article is to study the reliability analysis of the exponential model based on a new proposed symmetric loss function. The new proposed loss function is established on the basis of the LINEX asymmetric loss function. Firstly, the Bayes estimation of the parameter is derived under the prior distribution of the parameter based on non-information Quasi prior distribution, and then the admissibility of the estimators are also discussed. Furthermore, this paper puts forward a novel testing procedure to evaluate the lifetime performance of exponential products based on the new derived Bayes estimator. Finally, Monte Carlo statistical simulation and an applicable example are used to illustrate that the new proposed Bayes estimators and testing procedure are effective and feasible.
\end{abstract}

Keywords: exponential distribution; Bayes estimation; compound LINEX symmetric loss function; lifetime performance index

(Submitted on April 29, 2018; Revised on June 13, 2018; Accepted on July 26, 2018)

(C) 2018 Totem Publisher, Inc. All rights reserved.

\section{Introduction}

The exponential distribution is a very important lifetime distribution in lifetime and reliability studies. The statistical inference and application related to exponential distribution have received great attention. For example, Basak and Balakrishnan [1] studied the prediction problem of survival time of units from exponential distribution based on step-stress testing censored sample with the help of maximum likelihood (ML) approach. Xia et al. [2] put forward a novel likelihood ratio test on the basis of the Schwarz information criterion for detecting possible bathtub-shaped changes when facing a sequence of exponential distribution. Krishna and Goel [3] discussed the classical and Bayes estimation for exponential distribution under entropy loss function on the basis of randomly censored data. Based on a lifetime performance index, Wu et al. [4] designed two acceptance-sampling plans for the exponential model. Jana et al. [5] studied the Bayes reliability estimation for a stress-strength model related to exponential distribution. For more studies about exponential distribution one can see papers [6-10] and references therein.

Assume that $X$ is a product's lifetime, which follows an exponential distribution and has the following density function:

$$
f(x ; \theta)=\frac{1}{\theta} e^{-\frac{1}{\theta} x}, \quad x>0
$$

Here, $\theta>0$ is the unknown parameter.

Bayesian theory has been widely used in natural science, engineering technology, medicine, environmental science, insurance actuarial science, economy and other fields [11-14]. In Bayesian analysis, the loss function plays a critical role in the final estimation result. Because of the convenience of mathematical treatments, most Bayesian inference results are

* Corresponding author.

E-mail address: chaoshig@163.com 
studied under symmetric loss functions, such as the squared error loss function. However, in some situations, such as reliability and lifetime testing field, overestimation and underestimation often have different effects on decision results [15]. Then, the squared error loss function cannot work well. Asymmetric loss functions have been proposed by many scholars. LINEX loss is a well-known asymmetric loss function, and many Bayesian statistical inferences are studied under the LINEX loss function [16-19]. Though several symmetric and asymmetric loss functions have been put forward, it is still necessary for scholars to establish new loss functions [20-21]. Based on the LINEX loss function, Zhang [21] developed a new kind of symmetric loss function, named the compound LINEX symmetric loss function. He pointed out some excellent characteristics of the loss function and also discussed the Bayes estimation problem of parameters of normal distribution and exponential distribution under this loss function. In recent years, references [22-24] studied Bayesian statistical inferences of Poisson distribution, Pareto distribution, and Burr XII distribution under this compound LINEX loss function.

Inspired by reference [21], this paper will propose a new kind of compound LINEX symmetric loss function and then study the Bayesian reliability analysis of exponential distribution under the new proposed loss function.

It is very important for modern enterprises to assess the quality of the products effectively. Process capability indices (PCIs) are easy and effective measurement tools for assembling process performance and potential capability. Montgomery [25] introduced a special PCI $C_{L}$, to assess the products' lifetime performance whose life is the-larger-the-better. The index $C_{L}$ is called the lifetime performance index. This article will also study the Bayes estimation and testing procedure of $C_{L}$ for exponential products under the new proposed loss function.

The main study of this article is organized as follows:

Section 2 not only recalls some preliminary knowledge about prior distribution and $C_{L}$, but also constructs a new compound LINEX loss function. Furthermore, it discusses the properties of the loss function. Some preliminary knowledge about exponential distribution and the lifetime performance index will also be recalled. Section 3 studies the Bayesian estimation of the unknown parameter and $C_{L}$. Furthermore, a novel Bayes test of the lifetime performance index will be put forward. In Section 4, Monte Carlo simulations are utilized to demonstrate the performance of the ML estimator and the Bayes estimators, and an applied example shows the effectiveness of the Bayesian test procedure. Finally, Section 5 gives concluding remarks.

\section{Preliminary Knowledge}

\subsection{Prior Distribution}

Prior distribution is a key element in Bayesian inference. In most Bayesian statistics, it is often assumed that we can obtain some prior knowledge about the unknown parameter $\theta$ through investigating from past experience, simulation experiments, or experts' knowledge. Prior distribution is often used to model this prior knowledge.

Assume that the parameter $\theta$ has Quasi-prior distribution, which is a non-informative prior distribution with the following form:

$$
\pi(\theta) \propto \frac{1}{\theta^{d}}, \quad \theta>0
$$

Here, $d>0$ is the shape parameter of prior distribution (2). If $d=0$, then $\pi(\theta) \propto 1$ is a discrete prior distribution. If $d=1$, then $\pi(\theta) \propto 1 / \theta$ is a non-informative prior distribution.

\subsection{A New Compound LINEX Symmetric Loss Function}

As an important component in Bayesian analysis, choosing an appropriate loss function is an important topic. When one wants to estimate the reliability or failure rate, he/she will find that overestimates will bring greater losses than underestimates. Then, the development of new asymmetric loss functions is very necessary. The LINEX loss function is a very familiar asymmetric loss function, and it has the following form (Basu and Ebrahimi [26]):

$$
L_{c}(\Delta)=e^{c \Delta}-c \Delta-1, \quad c \neq 0
$$


Here, $\Delta=(\delta-\theta) / \theta$, and $\delta$ is an estimator of the parameter $\theta$.

In the following discussion, we will study the Bayes reliability analysis of exponential model under a new established symmetric loss function, which is a compound LINEX loss function. It is constructed as follows:

$$
L(\Delta)=L_{c}(\Delta)+L_{-c}(\Delta)=e^{c \Delta}+e^{-c \Delta}-2
$$

Where $\Delta=(\delta-\theta) / \theta$ and $c>0$ is the shape parameter of $L(\Delta)$. Figure 1 illustrates the shape of the new constructed loss function (4) with $c=1$.

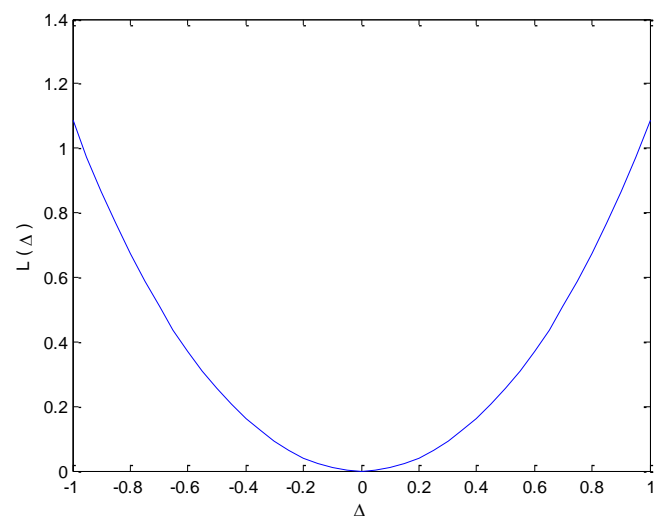

Figure 1. The shape of loss function $L(\Delta)$

From Figure 1 and the mathematical expression of the loss function (4), we know that the new constructed loss function (4) is a symmetric loss function.

Lemma 1 Let $\delta$ be an estimator of the parameter $\theta, \pi(\theta)$ is a prior distribution of parameter $\theta$, then under the new compound LINEX-based loss (4), the Bayes estimator of $\theta$ is the solution of equation (5), which has the following form:

$$
e^{-c} E\left(\frac{1}{\theta} e^{c / \delta} \mid X\right)=e^{c} E\left(\frac{1}{\theta} e^{-c / \delta} \mid X\right)
$$

The Bayes estimator is unique, when assuming the Bayes risk $r(\delta)<+\infty$.

Proof Under the new compound LINEX-based loss function (4), the Bayes risk of the estimator $\delta$ is:

$$
r(\delta)=E_{\theta}[E(L(\theta, \delta) \mid X)]
$$

To make the $r(\delta)$ minimum, we generally only need the minimum of $E[L(\theta, \delta) \mid X]$.

However,

$$
E[L(\theta, \delta) \mid X]=E\left[e^{c\left(\frac{\delta}{\theta}-1\right)}+e^{-c\left(\frac{\delta}{\theta}-1\right)}-2 \mid X\right]=e^{-c} E\left(\exp \frac{c \delta}{\theta} \mid X\right)+e^{c} E\left(\exp \frac{-c \delta}{\theta} \mid X\right)-2
$$

Therefore, we only need the right side of the upper formula to be minimal, as shown below:

$$
f(\delta)=e^{-c} E\left(\exp \frac{c \delta}{\theta} \mid X\right)+e^{c} E\left(\exp \frac{-c \delta}{\theta} \mid X\right)-2
$$


It is obvious that $f^{\prime \prime}(\delta)>0$, then the Bayes estimator $\hat{\delta}_{B}$ of parameter $\theta$ satisfies $f^{\prime}(\delta)=0$, i.e.,

$$
\frac{c}{\theta} \cdot e^{-c} E\left(\exp \frac{c \delta}{\theta} \mid X\right)+\frac{c}{\theta} \cdot e^{c} E\left(\exp \frac{-c \delta}{\theta} \mid X\right)=0
$$

Then, we obtain the result (5).

Now, we can prove the uniqueness by demonstrating that $r\left(\hat{\delta}_{B}\right)<+\infty$. According to the assumption $r(\delta)<+\infty$ and the definition of Bayes risk, we have $r\left(\hat{\delta}_{B}\right)<r(\delta)$. Then, $r\left(\hat{\delta}_{B}\right)<+\infty$.

\subsection{Lifetime Performance Index of Exponential Product}

The application and statistical inference of process capability indices have received great attention [27-30]. For many of the products, consumers want the life of the product to be as long as possible. Montgomery [25] proposed a special process capability index to assess the product, whose lifetime is the larger-the-better. The index is often called the lifetime performance index and has received widespread attention [31-34]. It is defined as

$$
C_{L}=\frac{\mu-L}{\sigma}
$$

Here, $L$ is the lower bound of the specifications.

Suppose that $X$ is the lifetime of a product, and it is distributed with exponential distribution (1). Then, we can get $\mu=E X=\theta$ and $\sigma=\sqrt{\operatorname{Var}(X)}=\theta$. Now, we can rewrite the index $C_{L}$ of exponential product as follows:

$$
C_{L}=\frac{\mu-L}{\sigma}=\frac{\theta-L}{\theta}=1-\frac{L}{\theta}
$$

The failure rate function $r(x)$ can be derived as

$$
r(x)=\frac{f(x ; \theta)}{1-F(x ; \theta)}=\frac{\theta^{-1} \exp \left(-\frac{x}{\theta}\right)}{\exp \left(-\frac{x}{\theta}\right)}=\frac{1}{\theta}
$$

From (11) and (12), we know that a larger $1 / \theta$ corresponds to a larger $C_{L}$ and a smaller $r(x)$. Therefore, $C_{L}$ can be a reasonable and accurate representative tool to assess the product performance.

A conforming rate is an important tool in measuring the product performance, and it is defined as

$$
P_{r}=P(X \geq L)
$$

For exponential distribution, we can get

$$
P_{r}=\int_{L}^{\infty} \theta^{-1} e^{-\frac{x}{\theta}} \mathrm{d} x=e^{C_{L}-1},-\infty<C_{L}<1
$$

According to equation (14), we can observe that $C_{L}$ and $P_{r}$ have a strictly one-to-one increasing relationship. When given the value of $C_{L}$, we can easily compute $P_{r}$ through equation (14), since there exists a one-to-one mapping between $P_{r}$ and $C_{L}$. Therefore, $C_{L}$ not only is a flexible tool to evaluate products' performance, but also is an effective tool to estimate the conforming rate of a product. 


\section{Bayes Reliability Analysis of Exponential Distribution}

Theorem 1 Assume that $X=\left(X_{1}, X_{2}, \cdots, X_{n}\right)$ is a sample drawn from exponential distribution (1), and parameter $\theta$ has the Quasi-prior (2). The symbol $x$ is the observation of $X$, and $x=\left(x_{1}, x_{2}, \cdots, x_{n}\right)$. Then, under the new compound LINEX-based loss function (4), the Bayes estimator of the unknown parameter $\theta$ is

$$
\hat{\theta}_{B}=\frac{2}{c}\left[\frac{1}{1+\exp (-2 c /(n+d))}-\frac{1}{2}\right] \cdot T
$$

Where $T=\sum_{i=1}^{n} X_{i}$

Proof Given sample observation $x=\left(x_{1}, x_{2}, \cdots, x_{n}\right)$, the likelihood function of parameter $\theta$ is

$$
L(x ; \theta)=\prod_{i=1}^{n} f\left(x_{i} ; \theta\right)=\prod_{i=1}^{n} \theta^{-1} \exp \left(-\theta^{-1} x_{i}\right)=\theta^{-n} e^{-t / \theta}
$$

Here, $t=\sum_{i=1}^{n} x_{i}$.

Then, the maximum likelihood estimator (MLE) of $\theta$ is

$$
\hat{\theta}_{M L}=\frac{T}{n}
$$

According to equation (16) and the Bayes' Theorem, we can derive the posterior probability density function of parameter $\theta$ as follows:

$$
h(\theta \mid x) \propto L(x ; \theta) \cdot \pi(\theta) \propto \theta^{-n} e^{-t / \theta} \cdot \theta^{-d} \propto \theta^{-(n+d)} e^{-t / \theta}
$$

Therefore, $h(\theta \mid x)$ is the density function of the inverse Gamma distribution, noted by $I \Gamma(n+d-1, t)$. That is,

$$
h(\theta \mid x)=\frac{t^{n+d-1}}{\Gamma(n+d-1)} \theta^{-(n+d)} e^{-\frac{t}{\theta}}
$$

Then, we have

$$
E\left[\frac{1}{\theta} \exp \left(\frac{c \delta}{\theta}\right) \mid X\right]=\int_{0}^{\infty} \frac{1}{\theta} \exp \left(\frac{c \delta}{\theta}\right) \cdot h(\theta \mid X) \mathrm{d} \theta=\frac{(n+d-1) T^{n+d-1}}{(T-c \delta)^{n+d}}
$$

and

$$
E\left[\frac{1}{\theta} \exp \left(\frac{-c \delta}{\theta}\right) \mid X\right]=\int_{0}^{\infty} \frac{1}{\theta} \exp \left(\frac{-c \delta}{\theta}\right) \cdot h(\theta \mid X) \mathrm{d} \theta=\frac{(n+d-1) T^{n+d-1}}{(T+c \delta)^{n+d}}
$$

Using equations (20), (21) and (5), we can solve the Bayes estimator of parameter $\theta$ as

$$
\hat{\theta}_{B}=\frac{2}{c}\left[\frac{1}{1+\exp (-2 c /(n+d))}-\frac{1}{2}\right] \cdot T
$$


Where $T=\sum_{i=1}^{n} X_{i}$.

Remark 1 By equation (11), the Bayesian estimator of $C_{L}$ is

$$
\widehat{C}_{B}=1-L / \hat{\theta}_{B}
$$

Then, to determine whether the products' lifetime meets a required level, this section will put forward a Bayes testing procedure for $C_{L}$.

We need to construct the following hypothesis at first:

$$
H_{0}: C_{L} \leq c_{0} \leftrightarrow H_{1}: C_{L}>c_{0}
$$

Let $Y=2 \theta^{-1} T \mid X$, then for given significance level $\alpha$, according to equation (12), we can easily show that $\theta^{-1} \mid X \sim \Gamma(n+d-1, T)$. Then, $Y \sim \chi^{2}(2(n+d-1))$. Let $\chi_{1-\alpha}^{2}(2(n+d-1))$ be the $1-\alpha$ percentile of $\chi^{2}(2(n+d-1))$. Then,

$$
P\left(2 \theta^{-1} T \leq \chi_{1-\alpha}^{2}(2(n+d-1)) \mid X\right)=1-\alpha
$$

That is,

$$
\begin{gathered}
P\left(\theta^{-1} \leq \frac{\chi_{1-\alpha}^{2}(2(n+d-1))}{2 T} \mid X\right)=1-\alpha \\
P\left(1-\theta^{-1} L \geq 1-L \cdot \frac{\chi_{1-\alpha}^{2}(2(n+d-1))}{2 T} \mid X\right)=1-\alpha
\end{gathered}
$$

Then, the lower credible limit of $C_{L}$ with significance level $\alpha$ can be derived as

$$
\underline{L B}=1-\left(1-\widehat{C}_{B}\right) \cdot \hat{\theta}_{B} \frac{\chi_{1-\alpha}^{2}(2(n+d-1))}{2 T}
$$

Now, we propose the Bayes testing procedure of $C_{L}$ as follows:

(i) For given sample size $n$, under the compound LINEX symmetric entropy loss function (5), calculate the Bayesian estimator $\hat{\theta}_{B}$ where $T=\sum_{i=1}^{n} X_{i}$.

(ii) Calculate the $1-\alpha$ one-side credible interval $[\underline{L B}, \infty)$ for lifetime performance index $C_{L}$, where $\underline{L B}$ is given in equation (28).

(iii) The rule of the Bayes testing procedure is given as follows:

If $c_{0} \notin[\underline{\mathrm{LB}}, \infty)$, then we reject $H_{0}$ and conclude that the products' lifetime adheres to the required level. Otherwise, we accept $H_{0}$ and conclude that the products' lifetime does not adhere to the required level.

\section{Examples}

Example 1 (Monte Carlo Simulations) Using the Monte Carlo statistical simulation, we use Matlab software to generate random samples drawn from the exponential distribution (1) with parameter $\theta=1.0$ and sample sizes $n=10,20,50,75,100$, 
respectively. Repeat the simulation experiment $N=5000$ times and use the mean $\hat{\theta}=\frac{1}{N} \sum_{i=1}^{N} \hat{\theta}_{i}$ as the estimated value of $\theta$, and use the mean square error $E R(\hat{\theta})=\frac{1}{N} \sum_{i=1}^{N}\left(\hat{\theta}_{i}-\theta\right)^{2}$ as a measure of good estimation standards. Here, $\hat{\theta}_{i}$ is the $i$ th estimate of the parameter $\theta$. The estimates and mean square errors of MLE and Bayes estimators are shown in Tables 1 and 2 under different sample sizes for $d=0$ and 1 . The mean square errors are shown in parentheses.

Table 1. Estimates and mean square errors under different sample sizes $(d=0)$

\begin{tabular}{|c|c|c|c|c|c|}
\multicolumn{7}{c}{ Table 1. Estimates and mean square errors under different sample sizes $(d=0)$} \\
\begin{tabular}{|c|c|c|c|c|c|}
\hline$n$ & 10 & 20 & 50 & 75 & 100 \\
\hline$\hat{\theta}_{M L}$ & $0.9978(0.0974)$ & $0.9974(0.0492)$ & $1.0010(0.0201)$ & $1.0026(0.0131)$ & $0.9981(0.0098)$ \\
\hline$\hat{\theta}_{B}(c=0.5)$ & $0.9969(0.0973)$ & $0.9972(0.0492)$ & $1.0010(0.0201)$ & $1.0026(0.0131)$ & $0.9980(0.0098)$ \\
\hline$\hat{\theta}_{B}(c=1.0)$ & $0.9945(0.0968)$ & $0.9966(0.0491)$ & $1.0009(0.0201)$ & $1.0025(0.0131)$ & $0.9980(0.0098)$ \\
\hline$\hat{\theta}_{B}(c=1.5)$ & $0.9904(0.0961)$ & $0.9956(0.0490)$ & $1.0007(0.0201)$ & $1.0025(0.0131)$ & $0.9980(0.0098)$ \\
\hline
\end{tabular}
\end{tabular}

Table 2. Estimates and mean square errors under different sample sizes $(d=1)$

\begin{tabular}{|c|c|c|c|c|c|}
\hline$n$ & 10 & 20 & 50 & 75 & 100 \\
\hline$\hat{\theta}_{M L}$ & $1.0029(0.0997)$ & $0.9975(0.0492)$ & $0.9973(0.0198)$ & $0.9986(0.0128)$ & $1.0010(0.0102)$ \\
\hline$\hat{\theta}_{B}(c=0.5)$ & $0.9111(0.0902)$ & $0.9498(0.0471)$ & $0.9777(0.0196)$ & $0.9855(0.0127)$ & $0.9910(0.0101)$ \\
\hline$\hat{\theta}_{B}(c=1.0)$ & $0.9092(0.0902)$ & $0.9493(0.0471)$ & $0.9776(0.0196)$ & $0.9854(0.0127)$ & $0.9910(0.0101)$ \\
\hline$\hat{\theta}_{B}(c=1.5)$ & $0.9061 \quad(0.0902)$ & $0.9484(0.0471)$ & $0.9774(0.0196)$ & $0.9853(0.0127)$ & $0.9910(0.0101)$ \\
\hline
\end{tabular}

From Tables 1 and 2, we can observe that Bayes estimators under compound LINEX-based loss function are affected by the values of $c$. When sample size $n$ is small, the value of parameter $c$ has a large influence on the estimation results; however, with an increase of $n$, the values of the mean square error decrease. When $n$ is larger than 50 , the influence of it is very small, and sometimes it can be ignored. The Bayes estimate is closer to the true value of $\theta$ as $n$ increases. At the same time, we find that when $n$ is large, the change of prior distribution has less influence on the estimation result.

Example 2 (An Application Example) To show the effectiveness and practicable properties of the new Bayes testing procedure, a practical example proposed in Nelson [35] is adopted. Under constant voltage stress, a life testing experiment is done on specimens of a type of electrical insulating fluid. The data set is reported in Table 3.

Table 3. Life testing experiment data

\begin{tabular}{|c|c|c|c|c|c|c|c|c|c|}
\hline \multicolumn{10}{|c|}{ Data Set $(n=19)$} \\
\hline 0.19 & 0.78 & 1.31 & 2.78 & 0.96 & 4.15 & 12.06 & 6.50 & 31.75 & 3.16 \\
\hline 4.85 & 72.89 & 32.52 & 4.67 & 7.35 & 8.27 & 8.01 & 33.91 & 36.71 & \\
\hline
\end{tabular}

Balakrishnan et al. [36] proved that the life testing data distributed with exponential distribution (1) by using the goodness of fit test combined with the least squares method. We will use this example to illustrate the steps of the new testing procedure of $C_{L}$, as follows:

(i) Determine the lower bound $L=1.04$. That is to say if an electrical insulting fluid's lifetime exceeds $L$ hours, then it is a conforming product. To satisfy the regarding operational performance, $P_{r}$ is required to exceed some value. Here, we assume the value is $80 \%$. According to equation (9), we know that the value of $C_{L}$ needs to exceed 0.80 . Then, we get the target value $c_{0}=0.80$.

(ii) Establish the following testing hypothesis

$$
H_{0}: C_{L} \leq 0.80 \leftrightarrow H_{1}: C_{L}>0.80
$$

(iii) Specify a significance level $\alpha=0.05$. 
(iv) According to equation (28), calculate the $95 \%$ credible interval for $C_{L}$.

Here, we assume parameter $\theta$ has the non-informative prior, i.e., $d=1$. For the loss function (4), the value of the shape parameter is $c=1.0$. Then, we can obtain $[\underline{\mathrm{LB}}, \infty)=[0.8982, \infty)$.

(v) Because $c_{0}=0.80 \notin[\underline{\mathrm{LB}}, \infty)$, then we reject $H_{0}$ and conclude that the products' lifetime meets the required level.

\section{Conclusions}

This paper first develops a symmetric loss function based on the most famous asymmetric LINEX loss function. Then, using the Quasi-prior distribution and the new proposed compound LINEX-based loss function, a Bayes estimator of unknown parameter of the exponential distribution is first derived and then a Bayes estimator of lifetime performance index $C_{L}$ is also obtained. Furthermore, this paper puts forward a new Bayes testing procedure of $C_{L}$ utilized to evaluate the products' lifetime performance. Application example shows that the new Bayes testing procedure of $C_{L}$ is easy and effective in evaluating whether the products' lifetime meets the required level.

\section{Acknowledgements}

The author, Haiping Ren, is thankful for the support of the National Natural Science Foundation of China (No. 71661012), the National Higher Vocational Language Teaching Reform Project of China (No. 201641), the PhD Research Startup Foundation of JXUST, and the Foundation of Educational Commission of Jiangxi Province (No. GJJ170496).

\section{References}

1. I. Basak and N. Balakrishnan, "Prediction of Censored Exponential Lifetimes in a Simple Step-Stress Model under Progressive Type II Censoring," Computational Statistics, Vol. 32, No. 4, pp. 1665-1687, December 2017

2. C. Xia, K. K. Said, and N. Wei, "Change-Point Analysis with Bathtub Shape for the Exponential Distribution," Journal of Applied Statistics, Vol. 43, No. 15, pp. 2740-2750, February 2016

3. H. Krishna and N. Goel, "Classical and Bayesian Inference in Two Parameter Exponential Distribution with Randomly Censored Data," Computational Statistics, Vol. 33, No. 2, pp. 1-27, March 2018

4. C. W. Wu, M. H. Shu, and Y. N. Chang, "Variable-Sampling Plans based on Lifetime-Performance Index under Exponential Distribution with Censoring and its Extensions," Applied Mathematical Modeling, Vol. 55, pp. 81-93, March 2018

5. N. Jana, S. Kumar, and K. Chatterjee, "Bayes Estimation for Exponential Distributions with Common Location Parameter and Applications to Multi-State Reliability Models," Journal of Applied Statistics, Vol. 43, No.15, pp. 2697-2712, February 2016

6. P. B. Nowak, "The MLE of the Mean of the Exponential Distribution based on Grouped Data is Stochastically Increasing," Statistics \& Probability Letters, Vol. 111, pp. 49-54, April 2016

7. J. H. Gove, “A Demographic Study of the Exponential Distribution Applied to Uneven-Aged Forests," Forestry, Vol. 90, No.1, pp. 18-31, January 2017

8. H. M. Barakat, E. M. Nigm, M. E. El-Adll, and M. Yusuf, "Prediction of Future Generalized Order Statistics based on Exponential Distribution with Random Sample Size,” Statistical Papers, Vol. 59, No. 2, pp.605-631, June 2018

9. H. S. Mohammed, S. F. Ateya, and E. K.Al-Hussaini, "Estimation based on Progressive First-Failure Censoring from Exponentiated Exponential Distribution,” Journal of Applied Statistics, Vol. 44, No. 8, pp. 1479-1494, July 2017

10. E. Isogai and C. Uno, "Three-Stage Confidence Intervals for a Linear Combination of Locations of Two Negative Exponential Distributions," Metrika, Vol. 81, No. 1, pp. 1-19, January 2018

11. J. H. Wang, W. Y. Yan, H. Xu, Y. Zhi, Z. R. Wang, and J. C. Jiang, "Investigation of the Probability of a Safe Evacuation to Succeed in Subway Fire Emergencies based on Bayesian Theory," Ksce Journal of Civil Engineering, Vol. 22, No. 3, pp. 877886, May 2018

12. L. A. Barnes, "Fine-Tuning in the Context of Bayesian Theory Testing," European Journal for Philosophy of Science, Vol. 8, No. 2, pp. 253-269, May 2018

13. Y. K. Gu, Y. G. Xiong, and J. Li, "A Reliability Analysis of Crank Rod System based on Bayesian Network," Nonferrous Metals Science \& Engineering, Vol. 3, No. 2, pp. 96-100, April 2012

14. P. Mehta, M. Kuttolamadom, and L. Mears, "Mechanistic Force Model for Machining Process-Theory and Application of Bayesian Inference," International Journal of Advanced Manufacturing Technology, Vol. 91, No. 9-12, pp. 3673-3682, August 2017

15. Z. Porosiński, "On Robust Bayesian Estimation under Some Asymmetric and Bounded Loss Function,” Statistics, Vol. 43, No. 3, pp. 253-265, May 2009

16. P. J. Williams and M. B. Hooten, "Combining Statistical Inference and Decisions in Ecology," Ecological Applications, Vol. 26, No. 6, pp. 1930-1942, March 2016

17. L. C. Hwang and C. H. Lee, "Bayes Sequential Estimation for a Poisson Process under a LINEX Loss Function," Statistics, Vol. 
47, No. 4, pp. 672-687, January 2013

18. A. O. A. Mutairi, "Bayesian Estimation using (Linex) for Generalized Power Function Distribution," Lobachevskii Journal of Mathematics, Vol. 39, No. 3, pp. 297-303, April 2018

19. A. T. P. Najafabadi and M. O. Najafabadi, "On the Bayesian Estimation for Cronbach's Alpha," Journal of Applied Statistics, Vol. 43, No. 13, pp. 2416-2441, March 2016

20. H. P. Ren, "Bayesian Estimation of Parameter of Rayleigh Distribution under Symmetric Entropy Loss Function," Journal of Jiangxi University of Science \& Technology, Vol. 31, No. 5, pp. 64-66, October 2010

21. R. Zhang, "The Parameter Estimation under the Compound LINEX Symmetric Loss Function," Dalian University of Technology, Dalian, 2010

22. C. D. Wei, S. Wei, and H. Su, "Bayes Estimation and Application of Poisson Distribution Parameter under Compound LINEX Symmetric Loss," Statistics and Decision, No.7, pp. 156-157, April 2010

23. C. D. Wei, S. Wei, and H. Su, "E-Bayes Estimation of Shape Parameter of Pareto Distribution under Compound LINEX Symmetric Loss and its Application," Statistics and Decision, No. 17, pp. 7-9, September 2009

24. S. Wei and Z. N. Li, "Bayes Estimation of Burr XII Distribution Parameter in the Composite LINEX Loss of Symmetry," Applied Mathematics A Journal of Chinese Universities, Vol. 32, No. 1, pp. 49-54, January 2017

25. D. C. Montgomery, "Introduction to Statistical Quality Control," John Wiley \& Sons, New York, 1985

26. A. Basu and N. Ebrahimi, "Bayesian Approach to Life Testing and Reliability Estimation using Asymmetric Loss Function," Journal of Statistical Planning \& Inference, Vol. 29, No.1, pp. 21-31, September 1991

27. A. Lepore, B. Palumbo, and P. Castagliola, "A Note on Decision Making Method for Product Acceptance based on Process Capability Indices Cpk, and Cpmk," European Journal of Operational Research, Vol. 267, No. 1, pp. 393-398, May 2018

28. M. Chatterjee and A. K. Chakraborty, "Unification of Some Multivariate Process Capability Indices for Asymmetric Specification Region,” Statistica Neerlandica, Vol. 71, No. 4, pp. 286-306, November 2017

29. S. Ş. Vahaplar and Ege Oruç Özlem, "A Modified Approach for Estimating Process Capability Indices using Improved Estimators," Pakistan Journal of Statistics, Vol. 33, No. 6, pp. 411-418, November 2017

30. P. Chen and Z. S. Ye, "A Systematic Look at the Gamma Process Capability Indices," European Journal of Operational Research, Vol. 265, No. 2, pp. 589-597, March 2018

31. S. F. Wu and M. J. Lin, "Computational Testing Algorithmic Procedure of Assessment for Lifetime Performance Index of Products with Weibull Distribution under Progressive Type I Interval Censoring," Journal of Computational and Applied Mathematics, Vol. 311, pp. 364-374, February 2017

32. S. F. Wu, "The Performance Assessment on the Lifetime Performance Index of Products Following Chen Lifetime Distribution based on the Progressive Type I Interval Censored Sample," Journal of Computational \& Applied Mathematics, Vol. 334, pp. 27-38, May 2018

33. S. Asghari, B. S. Gildeh, J. Ahmadi, and G. M. Borzadaran, "Lifetime Performance Index based on Ranked Set Sampling," Communication in Statistics- Simulation and Computation, Vol. 46, No. 9, pp. 7405-7422, April 2017

34. S. Dey, V. K. Sharma, M. Z. Anis, and B. Yadav, "Assessing Lifetime Performance Index of Weibull Distributed Products using Progressive Type II Right Censored Samples," International Journal of System Assurance Engineering \& Management, Vol. 8, No. 2, pp. 318-333, June 2017

35. J. F. Lawless, "Statistical Model and Methods for Lifetime Data," John Wiley \& Sons, New York, 1982

36. N. Balakrishnan, C. T. Lin, and P. S. Chan, "A Comparison of Two Simple Prediction Intervals for Exponential Distribution," IEEE Transactions on Reliability, Vol. 54, pp. 27-33, January 2005 